\title{
Locally unbounded topological fields with topological nilpotents
}

by

\author{
J. E. Marcos (Valladolid)
}

\begin{abstract}
We construct some locally unbounded topological fields having topologically nilpotent elements; this answers a question of Heine. The underlying fields are subfields of fields of formal power series. In particular, we get a locally unbounded topological field for which the set of topologically nilpotent elements is an open additive subgroup. We also exhibit a complete locally unbounded topological field which is a topological extension of the field of $p$-adic numbers; this topological field is a missing example in the classification of complete first countable fields given by Mutylin.
\end{abstract}

1. Introduction. A topological ring $(R, \mathcal{T})$ is a ring $R$ provided with a topology $\mathcal{T}$ such that the algebraic operations $(x, y) \mapsto x \pm y$ and $(x, y) \mapsto x y$ are continuous. A topological field $(K, \mathcal{T})$ is a field $K$ equipped with a ring topology $\mathcal{T}$ such that the inversion $x \mapsto x^{-1}$ is also continuous. For an introduction to topological fields, the books $[10,12,13]$ are recommended.

We recall that a subset $S$ of a commutative topological ring $R$ is bounded if given any neighborhood $V$ of zero, there exists a neighborhood $U$ of zero such that $S U \subseteq V$. If $R$ is a nondiscretely topologized field, this is equivalent to saying that given any neighborhood $V$ of zero, there exists a nonzero element $x \in R$ such that $S x \subseteq V$ (see [1, p. 94], [10, Theorem 3, p. 42] or [13, Lemma 12, p. 26]). A ring topology on $R$ is locally bounded if there is a bounded neighborhood of zero. Each field topology induced by a norm is locally bounded. An element $x$ of a topological ring is called topologically nilpotent if the sequence $\left(x^{n}\right)_{n \in \mathbb{N}}$ converges to zero.

In $[5,6]$ Heine wrote: "I do not know whether there exist locally unbounded topological fields which admit topologically nilpotent elements". We construct some topological fields satisfying both conditions. The underlying fields are subfields of some fields $K((X))$ of formal power series. In some cases we get locally unbounded topological fields in which the set of topological nilpotents is an open additive subgroup. This complements

2000 Mathematics Subject Classification: Primary 12J99. 
the topological characterization of normed fields by Cohn [3] (see Theorem 4.3 below). Our construction is somewhat similar to the topological fields given in [2, Lemma 3], but the latter are normed fields, and therefore locally bounded. The reader may also compare our examples with the locally unbounded topologies given by Gould [4].

In Section 5, we exhibit a complete locally unbounded topological field which is a topological extension of the $p$-adic number field $\mathbb{Q}_{p}$. This is a missing example in the classification of complete first countable topological fields given by Mutylin [9, Table 1] (see also [13, p. 256]). In [8] Mutylin gave an example of a locally bounded nonnormable field extension of the $p$-adic number field.

We recall that for a family $\left\{U_{i}\right\}_{i \in I}$ of subsets of a commutative ring $R$ to be a fundamental system of neighborhoods of zero for a Hausdorff ring topology $\mathcal{T}$ on $R$, it suffices that the following properties hold:

(1) For all $i \in I, 0 \in U_{i}, U_{i}=-U_{i}$.

(2) For all $i \in I$ there exists $k \in I$ such that $U_{k}+U_{k} \subseteq U_{i}$.

(3) For all $i \in I$ there exists $k \in I$ such that $U_{k} U_{k} \subseteq U_{i}$.

(4) For all $i \in I$ and $x \in R$ there exists $k \in I$ such that $x U_{k} \subseteq U_{i}$.

$$
\bigcap_{i \in I} U_{i}=\{0\} \text {. }
$$

If, in addition, $R$ is a field, then $\mathcal{T}$ is a field topology if $\left\{U_{i}\right\}_{i \in I}$ also satisfies the following condition:

(6) For all $i \in I$ there exists $k \in I$ such that $\left(1+U_{k}\right)^{-1} \subseteq 1+U_{i}$.

See [7], [10, p. 4], [12, p. 79] or [13, p. 3], for instance.

Throughout this paper, log denotes the natural logarithm.

2. A size function on a field. To get some bounds for defining certain subrings and subfields of fields of formal power series, we make the following definition.

Definition 2.1. Let $K$ be a field. We say that a function $N: K \rightarrow \mathbb{R}_{\geq 0}$ is a size function if the following conditions are satisfied:

(N1) $\quad N(a) \geq 2$ for all $a \in K$, and $N(0)=N(1)=N(-1)=2$.

(N2) $\quad N(a \pm b) \leq N(a)+N(b)$ for all $a, b \in K$.

(N3) $\quad N(a b) \leq N(a)+N(b)$ for all $a, b \in K$.

(N4) For each $m \in \mathbb{N}$ there exists $a \in K$ such that $N(a) \geq m$.

In some cases we will consider the following stronger conditions:

$N(a \pm b) \leq \max \{N(a), N(b)\}$ for all $a, b \in K$.

$\left(\mathrm{N}^{\prime}\right) \quad N(a b) \leq \max \{N(a), N(b)\}$ for all $a, b \in K$. 
If both $\left(\mathrm{N}^{\prime}\right)$ and $\left(\mathrm{N}^{\prime}\right)$ are satisfied, then the set $\{a \in K: N(a) \leq m\}$ is a subring of $K$ for each $m \geq 2$ (see [2] and Example 4). In this case, (N1) can be replaced by

$\left(\mathrm{N}^{\prime}\right) \quad N(a) \geq 1$ for all $a \in K$, and $N(0)=N(1)=N(-1)=1$.

Notice that $\log (x+y) \leq \log (x)+\log (y)$ for all $x \geq 2, y \geq 2$. Consequently, $\log (N(a+b)) \leq \log (N(a))+\log (N(b))$ and $\log (N(a b)) \leq \log (N(a))+$ $\log (N(b))$ for all $a, b \in K$.

We will give some examples for which it is easy to verify that conditions (N1)-(N4) are satisfied.

EXAMPLE 1. Let $k(X)$ be a field of rational functions. If $P(X), Q(X) \in$ $k[X]$ and $P(X) / Q(X)$ is an irreducible fraction we define

$$
N(P(X) / Q(X))=\max \{\operatorname{deg}(P(X)), \operatorname{deg}(Q(X)), 2\} .
$$

We can also define another size: $N_{\mathrm{d}}(P(X) / Q(X))=\max \{\operatorname{deg}(Q(X)), 2\}$.

ExAmple 2. Let $\mathbb{Q}$ be the field of rational numbers. Let $a / b \in \mathbb{Q}$ be an irreducible fraction, $a, b \in \mathbb{Z}$. We define

$$
N(a / b)=\log (\max \{|a|,|b|\})+2 .
$$

In this situation, another size function is $N_{\mathrm{d}}(a / b)=\log (|b|)+2$.

EXAmple 3 . In the above situation, let $\mathbb{P}$ be the set of positive prime numbers. We define

$$
N_{\mathrm{p}}(a / b)=\max (\{p \in \mathbb{P}: p \text { divides } b\} \cup\{2\}) .
$$

EXAMPLE 4. If a field $F$ is the union of a strictly increasing sequence of subfields, $F=\bigcup_{n \in \mathbb{N}} F_{n}$ with $F_{n} \subset F_{n+1}$, then we define

$$
N(a)=\min \left\{n: a \in F_{n}\right\} .
$$

This function $N$ has been used in [2]. In Examples 3 and 4 the inequalities $\left(\mathrm{N} 2^{\prime}\right)$ and $\left(\mathrm{N} 3^{\prime}\right)$ are satisfied.

Let $K$ be a field. We recall that a norm is a function \|\|$: K \rightarrow \mathbb{R}_{\geq 0}$ such that

- $\|0\|=0$, and $\|x\|>0$ for all $x \neq 0$;

- $\|x\|=\|-x\|$;

- $\|x+y\| \leq\|x\|+\|y\|$

- $\|x y\| \leq\|x\| \cdot\|y\|$.

A norm is nonarchimedean if $\|x+y\| \leq \max \{\|x\|,\|y\|\}$.

ExAmple 5. Let $(K,\|\|)$ be a normed field such that, for each $m \in \mathbb{N}$, there exists $x \in K$ such that $\|x\| \geq m$. We define the size function

$$
N(x)=\log \left(\max \left\{\|x\|, e^{2}\right\}\right) .
$$

If the norm is nonarchimedean, then this size function satisfies $\left(\mathrm{N}^{\prime}{ }^{\prime}\right)$. 
ExAmPle 6 . Let $\overline{\mathbb{Q}}$ be the algebraic closure of the rational number field. For each $\alpha \in \overline{\mathbb{Q}} \backslash\{0\}$ we let $\operatorname{deg}(\alpha)$ be the degree of the minimal polynomial of $\alpha$ over $\mathbb{Q}$. We define

$$
N(\alpha)=\max \{\log (\operatorname{deg}(\alpha)), 2\} \quad \text { and } \quad N(0)=2 .
$$

3. Construction of a topological field. In this section we define a local subring of $K[[X]]$ and its quotient field. Afterwards, we define the corresponding ring and field topologies and we study their properties. Notice that if a sequence $\left(x_{n}\right)_{n \in \mathbb{N}}$ of real numbers satisfies $\lim _{n \rightarrow \infty} \log \left(x_{n}\right) / n=0$, then

$$
\lim _{n \rightarrow \infty} \frac{\log \left(n^{k} \max \left\{x_{i}: i=1, \ldots, n\right\}\right)}{n}=0 \quad \text { for each } k \in \mathbb{N} .
$$

LEMma 3.1. Let $K$ be a field with a size function $N$. The set

$$
A=\left\{\sum_{n=0}^{\infty} a_{n} X^{n}: a_{n} \in K, \lim _{n \rightarrow \infty} \frac{\log \left(N\left(a_{n}\right)\right)}{n}=0\right\}
$$

is a local subring of the ring $K[[X]]$ of formal series. Its maximal ideal is generated by $X$.

Proof. It is clear that $A$ is a proper subset of $K[[X]]$. Let $\alpha=\sum_{n=0}^{\infty} a_{n} X^{n}$, $\beta=\sum_{n=0}^{\infty} b_{n} X^{n} \in A$. It is easy to see that $\alpha+\beta \in A$. We consider their product $\alpha \beta=\sum_{n=0}^{\infty} c_{n} X^{n}$, where $c_{n}=\sum_{i+j=n} a_{i} b_{j}$. We have the bound $N\left(c_{n}\right) \leq 2(n+1) \max \left\{N\left(a_{i}\right), N\left(b_{j}\right): i, j \in\{0,1, \ldots, n\}\right\}$. Consequently, $\lim _{n \rightarrow \infty} \log \left(N\left(c_{n}\right)\right) / n=0$, and $\alpha \beta \in A$.

Let $\alpha=\sum_{n=0}^{\infty} a_{n} X^{n} \in A$ with $a_{0} \neq 0$. We are going to see that $\alpha^{-1} \in A$. Since $a_{0}^{-1} \alpha \in A$, we assume that $a_{0}=1$. We have $\alpha^{-1}=\sum_{n=0}^{\infty} b_{n} X^{n} \in$ $K[[X]]$, where $b_{0}=1$, and

We compute inductively

$$
b_{n}=-\sum_{\substack{i+j=n \\ 0 \leq j<n}} a_{i} b_{j} \quad \text { for } n \geq 1
$$

$$
\begin{aligned}
& b_{1}=-a_{1}, \\
& b_{2}=-a_{2}+a_{1}^{2}, \\
& b_{3}=-a_{3}+2 a_{2} a_{1}-a_{1}^{3}, \quad \ldots
\end{aligned}
$$

If $n \geq 2$, the coefficient $b_{n}$ is a sum of less than $n^{2}$ terms, each a product of up to $n$ coefficients from $\left\{a_{i}: i=1, \ldots, n\right\}$. Hence, $N\left(b_{n}\right) \leq n^{3} \max \left\{N\left(a_{i}\right)\right.$ : $i=0,1, \ldots, n\}$, and therefore $\lim \log \left(N\left(b_{n}\right)\right) / n=0$. We have proven that $\alpha^{-1} \in A$. We conclude that

$$
(X)=\left\{\sum_{n=1}^{\infty} a_{n} X^{n} \in A\right\}
$$

is the only maximal ideal of the ring $A$. 
We denote by $A^{\times}$the set of invertible elements of the ring $A$. A series $\sum_{n=0}^{\infty} a_{n} X^{n}$ is in $A^{\times}$if and only if $a_{0} \neq 0$.

Lemma 3.2. The ring $A$ is Noetherian. Its only nonzero ideals are $\left(X^{n}\right)$, $n \in \mathbb{N} \cup\{0\}$.

Proof. The first part is a consequence of the second. Let $I \neq A$ be a nontrivial ideal and let $\alpha=\sum_{n=m}^{\infty} a_{n} X^{n} \in I$ be a nonzero element with minimum additive $X$-adic valuation; that is, $v_{X}(\alpha)=m=\min \left\{v_{X}(\beta): \beta \in\right.$ $I \backslash\{0\}\}$. Since $\gamma=\sum_{n=m}^{\infty} a_{n} X^{n-m} \in A^{\times}$is a unit, we have $X^{m}=\alpha \gamma^{-1} \in I$. Now it is clear that $I=\left(X^{m}\right)$.

The quotient field of $A$ is

$$
E=\left\{\sum_{n=m}^{\infty} a_{n} X^{n}: m \in \mathbb{Z}, \lim _{n \rightarrow \infty} \frac{\log \left(N\left(a_{n}\right)\right)}{n}=0\right\} .
$$

For each $m \in \mathbb{N}$ we define a subset of $A$ :

$$
U_{m}=\left\{\sum_{n \geq m}^{\infty} a_{n} X^{n} \in A: \frac{\log \left(N\left(a_{n}\right)\right)}{n} \leq \frac{1}{m}\right\} .
$$

LEMMA 3.3. The family $\mathcal{B}=\left\{U_{m}\right\}_{m \in \mathbb{N}}$ is a neighborhood base at zero for a Hausdorff ring topology on A. Furthermore, the inversion is continuous in $A^{\times}$.

Proof. We check that $\mathcal{B}$ has properties (1)-(5). Properties (1) and (5) are trivial. It is easy to verify that $U_{2 m}+U_{2 m} \subseteq U_{m}$ for all $m \in \mathbb{N}$, and therefore condition (2) holds.

To check (3), for each $k \geq 2$, we take $m=4 k^{2} \geq 16$ and show that $U_{m} U_{m} \subseteq U_{k}$. Let $\alpha=\sum_{n=m}^{\infty} a_{n} X^{n}, \beta=\sum_{n=m}^{\infty} b_{n} X^{n} \in U_{m}$. Then $\alpha \beta=$ $\sum_{n=2 m}^{\infty} c_{n} X^{n}$, where $c_{n}=\sum_{i+j=n} a_{i} b_{j}$. Let

$$
r=\max \left\{N\left(a_{i}\right), N\left(b_{j}\right): i, j \in\{m, m+1, \ldots, n\}\right\} .
$$

We get the bound $N\left(c_{n}\right) \leq 2 n r$. Recalling that $\log (2 n) / n \leq 1 / \sqrt{n}$ for $n \geq 9$, we obtain

$$
\frac{\log \left(N\left(c_{n}\right)\right)}{n} \leq \frac{\log (2 n)}{n}+\frac{\log (r)}{n} \leq \frac{1}{\sqrt{n}}+\frac{1}{4 k^{2}} \leq \frac{1}{2 k}+\frac{1}{4 k^{2}} \leq \frac{1}{k} .
$$

So $\alpha \beta \in U_{k}$.

To prove (4), we first consider a polynomial $\beta=\sum_{n=0}^{t} b_{n} X^{n} \in K[X] \subset A$ and a neighborhood of zero $U_{m} \in \mathcal{B}$. Let $r=\max \left\{N\left(b_{n}\right): n=0, \ldots, t\right\}$. There exists $s \geq 3 m$ such that

$$
\frac{\log (r)}{n} \leq \frac{1}{3 m} \quad \text { and } \quad \frac{\log (t+1)}{n} \leq \frac{1}{3 m} \quad \text { for all } n \geq s .
$$

We now take the neighborhood $U_{s} \in \mathcal{B}$ and check that $\beta U_{s} \subseteq U_{m}$. Given $\alpha=\sum_{n=s}^{\infty} a_{n} X^{n} \in U_{s}$, we write $\beta \alpha=\sum_{n=0}^{\infty} c_{n} X^{n}$, where $c_{n}=\sum_{i=0}^{t} b_{i} a_{n-i}$ 
for $n \geq t$. Then

$$
N\left(c_{n}\right) \leq(t+1)\left(r+\max \left\{N\left(a_{i}\right): i=s, s+1, \ldots, n\right\}\right) .
$$

Applying (7), we get the bound

$$
\begin{aligned}
\frac{\log \left(N\left(c_{n}\right)\right)}{n} & \leq \frac{\log (t+1)}{n}+\frac{\log (r)}{n}+\frac{\log \left(\max \left\{N\left(a_{i}\right): i=s, \ldots, n\right\}\right)}{n} \\
& \leq \frac{1}{3 m}+\frac{1}{3 m}+\frac{1}{3 m}=\frac{1}{m} .
\end{aligned}
$$

Thus $\beta \alpha \in U_{m}$.

Second, let $\delta=\sum_{n=0}^{\infty} d_{n} X^{n} \in A$ be an arbitrary element, and $U_{m} \in \mathcal{B}$. We have seen that there exists $U_{l} \in \mathcal{B}$ such that $U_{l} U_{l} \subset U_{2 m}$. We split $\delta$ into a polynomial $\delta_{1}=\sum_{n=0}^{t} d_{n} X^{n}$ and $\delta_{2}=\sum_{n=t+1}^{\infty} d_{n} X^{n} \in U_{l}$. By the above we get a neighborhood $U_{s} \in \mathcal{B}$ (with $s \geq l$ ) such that $\delta_{1} U_{s} \subset U_{2 m}$. So

$$
\delta U_{s} \subseteq \delta_{1} U_{s}+\delta_{2} U_{s} \subseteq U_{2 m}+U_{l} U_{l} \subseteq U_{2 m}+U_{2 m} \subset U_{m} .
$$

To show that inversion is continuous in $A^{\times}$, it suffices to show that it is continuous at 1 (see [12, p. 106]). For this purpose, we now check that, given a zero neighborhood $U_{m} \in \mathcal{B}$, there exists $U_{k} \in \mathcal{B}$ such that $\left(1+U_{k}\right)^{-1} \subseteq$ $1+U_{m}$. We choose $k \geq 2 m$ such that $3 \log (n) / n \leq 1 /(2 m)$ for all $n \geq k$.

Let $\alpha=1+\sum_{n=k}^{\infty} a_{n} X^{n} \in 1+U_{k}$. Then $\alpha^{-1}=1+\sum_{n=k}^{\infty} b_{n} X^{n}$. Using the same reasoning as in the proof of Lemma 3.1, we get

$$
N\left(b_{n}\right) \leq n^{3} \max \left\{N\left(a_{i}\right): i=k, \ldots, n\right\} .
$$

Consequently,

$$
\begin{aligned}
\frac{\log \left(N\left(b_{n}\right)\right)}{n} & \leq \frac{3 \log (n)}{n}+\frac{\log \left(\max \left\{N\left(a_{i}\right): i=k, \ldots, n\right\}\right)}{n} \\
& \leq \frac{1}{2 m}+\frac{1}{k} \leq \frac{1}{m} .
\end{aligned}
$$

Thus $\alpha^{-1} \in 1+U_{m}$.

We denote this ring topology on $A$ by $\mathcal{T}_{N}$. It is clear that the maximal ideal $(X)$ is open. Notice that, for all $k, m \in \mathbb{N}$, the following inclusions hold:

$$
U_{m+k} \subset X^{k} U_{m} \subset U_{m}, \quad U_{m+k} \subset X^{-k} U_{m+k} \subset U_{m} .
$$

Lemma 3.4. The same family $\mathcal{B}=\left\{U_{m}\right\}_{m \in \mathbb{N}}$ is a neighborhood base at zero for a Hausdorff field topology on the quotient field $E$ of $A$. We also denote this topology by $\mathcal{T}_{N}$.

Proof. We have proven in the previous lemma that $\mathcal{B}$ has properties (1), (2), (3), (5) and (6). It remains to prove (4). We have also shown in the previous lemma that, for each $\delta \in A$ and each $U_{m} \in \mathcal{B}$, there exists $U_{s}$ such that $\delta U_{s} \subseteq U_{m}$. This fact together with (8) shows that property (4) holds in $E$. 
If condition $\left(\mathrm{N}^{\prime}\right)$ is satisfied, then the neighborhoods $U_{m}$ are open additive subgroups of $(A,+)$.

Lemma 3.5. The ring of polynomials $K[X]$ is a dense subring of $\left(A, \mathcal{T}_{N}\right)$.

Proof. Given $\alpha=\sum_{n=0}^{\infty} a_{n} X^{n} \in A$ and a zero neighborhood $U_{m} \in \mathcal{B}$, since

$$
\lim _{n \rightarrow \infty} \log \left(N\left(a_{n}\right)\right) / n=0,
$$

there exists $t \geq m$ such that $\log \left(N\left(a_{n}\right)\right) / n \leq 1 / m$ for all $n \geq t$. Hence $\alpha-\sum_{n=0}^{t-1} a_{n} X^{n} \in U_{m}$. Thus $\left(\alpha+U_{m}\right) \cap K[X] \neq \emptyset$.

We consider in $K[X]$ the subspace topology inherited from $\left(A, \mathcal{T}_{N}\right)$; we denote this topology also by $\mathcal{T}_{N}$. A fundamental system of zero neighborhoods is $\mathcal{B}^{\prime}=\left\{U_{m}^{\prime}\right\}_{m \in \mathbb{N}}$, where $U_{m}^{\prime}=U_{m} \cap K[X]$. We are going to see that $\left(A, \mathcal{T}_{N}\right)$ is the completion of the topological ring $\left(K[X], \mathcal{T}_{N}\right)$. We construct the completion as the quotient ring of the ring of Cauchy sequences by the ideal of all sequences converging to zero. We say that two Cauchy sequences are equivalent if they represent the same element in this quotient ring.

Lemma 3.6. Let $\left(\alpha_{m}\right)_{m \in \mathbb{N}}$ be a Cauchy sequence in $\left(K[X], \mathcal{T}_{N}\right)$. There exists a sequence $\left(b_{n}\right)_{n \geq 0}$ of elements of $K$ such that the sequence $\left(\omega_{m}\right)_{m \in \mathbb{N}}$ of polynomials defined by $\omega_{m}=\sum_{n=0}^{m} b_{n} X^{n}$ is a Cauchy sequence equivalent to $\left(\alpha_{m}\right)_{m \in \mathbb{N}}$.

Proof. Passing to subsequences we may assume that $\alpha_{i}-\alpha_{j} \in U_{m+1}^{\prime}$ for all $i, j \geq m$. We write $\alpha_{i}=\sum_{n=0}^{d_{i}} a_{i, n} X^{n}$. Hence $a_{i, n}=a_{j, n}$ for $i, j \leq n$, and we define $b_{n}=a_{n, n}$. Given a neighborhood $U_{m}^{\prime} \in \mathcal{B}^{\prime}$, for any index $k>\max \left\{\operatorname{deg}\left(\alpha_{2 m}\right), 2 m\right\}$, we take

$$
\alpha_{k}-\omega_{k}=\left(\alpha_{k}-\alpha_{2 m}\right)+\left(\alpha_{2 m}-\omega_{k}\right) .
$$

Notice that if $\sum_{n=s}^{t} c_{n} X^{n} \in U_{s}^{\prime}$ and $s \leq l<t$, then $\sum_{n=s}^{l} c_{n} X^{n} \in U_{s}^{\prime}$ as well. Since $\alpha_{k}-\alpha_{2 m} \in U_{2 m+1}^{\prime}$ and $k>\operatorname{deg}\left(\alpha_{2 m}\right)$, we see that also $\omega_{k}-\alpha_{2 m} \in U_{2 m+1}^{\prime}$. Using (9), we obtain $\alpha_{k}-\omega_{k} \in U_{2 m+1}^{\prime}+U_{2 m+1}^{\prime} \subset U_{m}^{\prime}$. Thus $\left(\omega_{m}\right)_{m \in \mathbb{N}}$ is a Cauchy sequence equivalent to $\left(\alpha_{m}\right)_{m \in \mathbb{N}}$.

Lemma 3.7. The topological ring $\left(A, \mathcal{T}_{N}\right)$ is a completion of $\left(K[X], \mathcal{T}_{N}\right)$.

Proof. We have seen in Lemma 3.5 that $K[X]$ is a dense subring of $\left(A, \mathcal{T}_{N}\right)$. Let $\left(\alpha_{m}\right)_{m \in \mathbb{N}}$ be a Cauchy sequence in $\left(K[X], \mathcal{T}_{N}\right)$. Using the previous lemma, we get an equivalent Cauchy sequence $\left(\omega_{m}\right)_{m \in \mathbb{N}}$ such that $\omega_{m}=\sum_{n=0}^{m} b_{n} X^{n}$. Given a neighborhood $U_{k}^{\prime} \in \mathcal{B}^{\prime}$, there exists $s \in \mathbb{N}$ such that $\omega_{i}-\omega_{j} \in U_{k}^{\prime}$ for all $i, j \geq s$. In particular, $\omega_{i}-\omega_{s} \in U_{k}^{\prime}$ for all $i \geq s$. Hence $\log \left(N\left(b_{n}\right)\right) / n \leq 1 / k$ for all $n \geq s$; that is, $\lim _{n \rightarrow \infty} \log \left(N\left(b_{n}\right)\right) / n=0$. Thus $\sum_{n=0}^{\infty} b_{n} X^{n} \in A$ is the limit in $\left(A, \mathcal{T}_{N}\right)$ of the sequence $\left(\omega_{m}\right)_{m \in \mathbb{N}}$.

Corollary 3.8. The topological ring $\left(A, \mathcal{T}_{N}\right)$ is complete. 
Corollary 3.9. The topological field $\left(E, \mathcal{T}_{N}\right)$ is complete.

Proof. Let $\left(\alpha_{m}\right)_{m \in \mathbb{N}}$ be a Cauchy sequence in $\left(E, \mathcal{T}_{N}\right)$. We take the neighborhood $U_{1} \in \mathcal{B}$. There exists $s \in \mathbb{N}$ such that $\alpha_{i}-\alpha_{j} \in U_{1}$ for all $i, j \geq s$. Since $U_{1} \subset A$, we have $\alpha_{i}-\alpha_{s} \in A$ for all $i \geq s$. The sequence $\left(\alpha_{i}-\alpha_{s}\right)_{i \geq s}$ is Cauchy in $\left(A, \mathcal{T}_{N}\right)$, hence it has a limit $\gamma$. Therefore $\gamma+\alpha_{s}$ is the limit of $\left(\alpha_{m}\right)_{m \in \mathbb{N}}$ in $\left(E, \mathcal{T}_{N}\right)$.

The topological field $\left(E, \mathcal{T}_{N}\right)$ is a completion of $K(X)$ endowed with the subspace topology. Let $R$ be a complete metrizable ring with identity. If $R^{\times}$ is open, then inversion is continuous [12, Theorem 14.10]. Since both $\left(A, \mathcal{T}_{N}\right)$ and $\left(E, \mathcal{T}_{N}\right)$ are Hausdorff and satisfy the first axiom of countability, both are metrizable. We deduce again that inversion is continuous in $A$ and $E$.

In order to prove our main result we need the following easy lemma.

LEMMA 3.10. Let $(F, \mathcal{T})$ be a locally bounded topological field with a topologically nilpotent element $b \in F \backslash\{0\}$. There exists a zero neighborhood $V$ such that $\left\{b^{k} V\right\}_{k \in \mathbb{N}}$ is a neighborhood base at zero for the topology $\mathcal{T}$.

Proof. Let $\mathcal{B}$ be a fundamental system of zero neighborhoods. For every $a \in F \backslash\{0\}$ and $U \in \mathcal{B}$ the set $a U$ is also a zero neighborhood. Let $V \in \mathcal{B}$ be a bounded neighborhood of zero. Hence $\{U V\}_{U \in \mathcal{B}}$ is another neighborhood base at zero. For each $U \in \mathcal{B}$ there exists $b^{k} \in U$, and so $b^{k} V \subset U V$. Since each $b^{k} V$ is a zero neighborhood, the collection $\left\{b^{k} V\right\}_{k \in \mathbb{N}}$ is a fundamental system of zero neighborhoods.

THEOREM 3.11. The topological field $\left(E, \mathcal{T}_{N}\right)$ is locally unbounded and has topologically nilpotent elements.

Proof. It is obvious that $X \in E$ is topologically nilpotent. Using the previous lemma, we only need to prove that for each $U_{m} \in \mathcal{B}$ the family $\left\{X^{k} U_{m}\right\}_{k \in \mathbb{N}}$ is not a neighborhood base at zero. For this purpose it suffices to show that $X^{k} U_{m} \nsubseteq U_{2 m}$ for all $k, m \in \mathbb{N}$. Assume that there are $s, m$ such that $X^{s} U_{m} \subseteq U_{2 m}$. Using property (N4), we get $a \in K \subset E$ and $n \in \mathbb{N}$ such that

$$
n \geq m, \quad n \geq 4 s, \quad \frac{3}{4 m}<\frac{\log (N(a))}{n} \leq \frac{1}{m} .
$$

Hence $a X^{n} \in U_{m}$, and $a X^{s+n} \in X^{s} U_{m} \subseteq U_{2 m}$. Since $n \geq 4 s$, we have $n+s \leq n+n / 4=5 n / 4$. Consequently,

$$
\frac{\log (N(a))}{n+s} \geq \frac{\log (N(a))}{5 n / 4}>\frac{3}{4 m} \cdot \frac{4}{5}=\frac{3}{5 m}>\frac{1}{2 m}
$$

Thus $a X^{s+n} \notin U_{2 m}$, and we have got a contradiction.

4. An additive subgroup consisting of topological nilpotents. In this section we deal with fields with a size function that satisfies condi- 
ton $\left(\mathrm{N} 2^{\prime}\right.$ ) (see Examples 3, 4, and Example 5 in case the norm is nonarchimedean). We will present a result slightly stronger than Theorem 3.11.

THEOREM 4.1. Let $K$ be a field with a size function $N$ that satisfies condition $\left(\mathrm{N}^{\prime}\right)$. The set of topologically nilpotent elements of the topological ring $\left(A, \mathcal{T}_{N}\right)$ is the maximal ideal $(X) \subset A$.

Proof. It is clear that if an element does not belong to $(X)$, then it is not topologically nilpotent. Let $\alpha=\sum_{n=1}^{\infty} a_{n} X^{n} \in(X)$. For each $s \in \mathbb{N}$ we take the power $\alpha^{s}=\sum_{n=s}^{\infty} b_{n, s} X^{n}$. Each coefficient $b_{n, s}$ is a sum of products of $s$ terms (possibly repeated) taken from $\left\{a_{1}, \ldots, a_{n-s+1}\right\}$. Applying condition $\left(\mathrm{N} 2^{\prime}\right)$, we obtain $N\left(b_{n, s}\right) \leq s \max \left\{N\left(a_{1}\right), \ldots, N\left(a_{n}\right)\right\}$, and hence

$$
\frac{\log \left(N\left(b_{n, s}\right)\right)}{n} \leq \frac{\log (s)+\log \left(\max \left\{N\left(a_{1}\right), \ldots, N\left(a_{n}\right)\right\}\right)}{n} .
$$

Given a neighborhood of zero $U_{m} \in \mathcal{B}$, since $\lim _{n \rightarrow \infty} \log \left(N\left(a_{n}\right)\right) / n=0$, there exists $n_{m} \geq m$ such that

$$
\frac{\log \left(\max \left\{N\left(a_{1}\right), \ldots, N\left(a_{n}\right)\right\}\right)}{n} \leq \frac{1}{2 m}
$$

for all $n \geq n_{m}$. We take $s_{m} \geq n_{m}$ such that $\log (s) / s \leq 1 /(2 m)$ for all $s \geq s_{m}$. Therefore

$$
\frac{\log (s)}{n} \leq \frac{1}{2 m}
$$

for all $n \geq s$ and $s \geq s_{m}$. From (10)-(12), we conclude that

$$
\frac{\log \left(N\left(b_{n, s}\right)\right)}{n} \leq \frac{1}{m}
$$

for all $s \geq s_{m}$ and $n \geq s$. Thus $\alpha^{s} \in U_{m}$ for all $s \geq s_{m}$. We have proved that $\alpha$ is topologically nilpotent.

COROLlary 4.2. The topological field $\left(E, \mathcal{T}_{N}\right)$ is locally unbounded and the set of topologically nilpotent elements is an open additive subgroup.

Since an open additive subgroup is a neighborhood of zero, our last result is in a sense complementary to the following result of Cohn:

Theorem 4.3 ([3], [11], [10, p. 66], [13, p. 74]). Let $\mathcal{T}$ be a proper ring topology on a field. The following statements are equivalent:

(1) $\mathcal{T}$ is induced by a norm.

(2) $\mathcal{T}$ is locally bounded and there exists a nonzero topologically nilpotent element.

(3) The set of topologically nilpotent elements is a bounded open neighborhood of zero.

The following formulation of Warner is more related to our result: 
THEOREM 4.4 ([12, p. 132]). Let $F$ be a nontrivial topological field. The following statements are equivalent:

(1) The set of topological nilpotents is a bounded open additive subgroup.

(2) The topology of $F$ is given by a spectral ultranorm.

\section{A locally unbounded complete extension of the $p$-adic number}

field. In [9, Table 1] Mutylin raised the question if there exists a complete, non-locally bounded and first countable field extension of the $p$-adic number field $\mathbb{Q}_{p}$ (see also $[13$, p. 256]). In this section we exhibit a topological field with the above features. We omit the proofs because they are completely analogous to the corresponding ones in the previous sections.

Let $P(X) \in \mathbb{Z}[X]$. Any greatest common divisor of the coefficients of $P(X)$ is called a content of $P(X)$. We write $c(P)$ for a content of $P(X)$. We fix a prime $p$ and define a local subring $S$ of $\mathbb{Q}(X)$ by

$$
S=\mathbb{Z}[X]_{(p)}=\left\{\frac{P(X)}{R(X)}: P(X), R(X) \in \mathbb{Z}[X] \text { and } p \nmid c(R)\right\} .
$$

That is, $S$ is the ring of polynomial fractions whose denominator has content coprime to $p$. We define the following ring of formal power series, where $N$ is the size function in $\mathbb{Q}(X)$ defined in Example 1:

$$
A_{p}=\left\{\sum_{n=0}^{\infty} f_{n}(X) p^{n}: f_{n}(X) \in S, \lim _{n \rightarrow \infty} \frac{\log \left(N\left(f_{n}\right)\right)}{n}=0\right\} .
$$

The sum and product are performed in the following way: Given

$$
\alpha=\sum_{n=0}^{\infty} f_{n}(X) p^{n}, \quad \beta=\sum_{n=0}^{\infty} g_{n}(X) p^{n}
$$

in $A_{p}$, we set

$$
\begin{aligned}
\alpha+\beta & =\sum_{n=0}^{\infty}\left(f_{n}(X)+g_{n}(X)\right) p^{n}, \\
\alpha \beta & =\sum_{n=0}^{\infty}\left(\sum_{i+j=n} f_{i}(X) g_{j}(X)\right) p^{n} .
\end{aligned}
$$

Notice that the representation of elements in $A_{p}$ is not unique. The ring $A_{p}$ is local with the maximal ideal $p A_{p}=\left\{\sum_{n=1}^{\infty} f_{n}(X) p^{n} \in A_{p}\right\}$. The field of fractions of $A_{p}$ is

$$
E_{p}=\left\{\sum_{n=0}^{\infty} f_{n}(X) p^{n}: \begin{array}{l}
f_{0}(X) \in \mathbb{Q}(X), \\
f_{n}(X) \in S \text { if } n \geq 1, \quad \lim _{n \rightarrow \infty} \frac{\log \left(N\left(f_{n}\right)\right)}{n}=0
\end{array},\right.
$$

where the operations are performed as in (13). We have the inclusions 
$\mathbb{Z}_{p}[X] \subset A_{p}, \mathbb{Q}_{p}(X) \subset E_{p}$. For each $m \in \mathbb{N}$ we define a subset of $A_{p}$ by

$$
W_{m}=\left\{\sum_{n \geq m}^{\infty} f_{n}(X) p^{n} \in A_{p}: \frac{\log \left(N\left(f_{n}\right)\right)}{n} \leq \frac{1}{m}\right\} .
$$

The collection $\mathcal{B}_{W}=\left\{W_{m}\right\}_{m \in \mathbb{N}}$ is a fundamental system of zero neighborhoods for a Hausdorff ring topology on $A_{p}$; furthermore the inversion is continuous in $A_{p}^{\times}$. This family $\mathcal{B}_{W}$ is also a fundamental system of zero neighborhoods for a Hausdorff field topology on $E_{p}$. We use the notation $\mathcal{T}_{W}$ for both topologies.

We denote by the same symbol $\mathcal{T}_{W}$ the subspace topology that $\mathbb{Q}(X)$ inherits from $\left(E_{p}, \mathcal{T}_{W}\right)$, as well as the subspace topology that the subring $S$ inherits from the two topological rings $\left(\mathbb{Q}(X), \mathcal{T}_{W}\right)$ and $\left(A_{p}, \mathcal{T}_{W}\right)$. The topological ring $\left(A_{p}, \mathcal{T}_{W}\right)$ is the completion of $\left(S, \mathcal{T}_{W}\right)$, and $\left(E_{p}, \mathcal{T}_{W}\right)$ is the completion of $\left(\mathbb{Q}(X), \mathcal{T}_{W}\right)$. Hence $\left(E_{p}, \mathcal{T}_{W}\right)$ is a complete topological field. In addition, following the same reasoning as in Section 3, we deduce that the topological field $\left(E_{p}, \mathcal{T}_{W}\right)$ is locally unbounded and has topologically nilpotent elements.

The subspace topology that the field $\mathbb{Q}_{p}$ of $p$-adic numbers inherits from $\left(E_{p}, \mathcal{T}_{W}\right)$ is just the $p$-adic topology $\mathcal{T}_{p}$. Hence we have the following result, which is an answer to the aforementioned question of Mutylin.

THEOREM 5.1. The topological field $\left(E_{p}, \mathcal{T}_{W}\right)$ is a complete first countable locally unbounded topological extension of the p-adic number field $\left(\mathbb{Q}_{p}, \mathcal{T}_{p}\right)$.

\section{References}

[1] V. I. Arnautov, S. T. Glavatsky and A. V. Mikhalev, Introduction to the Theory of Topological Rings and Modules, Dekker, New York, 1996.

[2] J.-A. Cohen, Norms on F(X), Pacific J. Math. 109 (1983), 81-87.

[3] P. M. Cohn, An invariant characterization of pseudo-valuations on a field, Proc. Cambridge Philos. Soc. 50 (1954), 159-177.

[4] G. G. Gould, Locally unbounded topological fields and box topologies on products of vector spaces, J. London Math. Soc. 36 (1961), 273-281.

[5] J. Heine, Existence of locally unbounded topological fields, and field topologies which are not the intersection of bounded ring topologies, ibid. 5 (1972), 481-487.

[6] - Ring topologies of type $N$ on fields, General Topology Appl. 3 (1973), 135-148.

[7] J. O. Kiltinen, Inductive ring topologies, Trans. Amer. Math. Soc. 134 (1968), 149169.

[8] A. F. Mutylin, A non-normable extension of the p-adic field, Math. Notes 2 (1967), 496-498 [Mat. Zametki 2 (1967), 11-14].

[9] - Connected, complete, locally bounded fields. Complete non-locally bounded fields, Math. USSR-Sb. 5 (1968), 433-449 [Mat. Sb. 76 (1968), 454-472].

[10] N. Shell, Topological Fields and Near Valuations, Dekker, New York, 1990.

[11] S. Singh, Note on a paper of P. M. Cohn, Math. Proc. Cambridge Philos. Soc. 78 (1975), 211-213. 
[12] S. Warner, Topological Fields, North-Holland, Amsterdam, 1989.

[13] W. Więsław, Topological Fields, Dekker, New York, 1988.

Departamento Algebra y Geometría

Facultad de Ciencias

47005 Valladolid, Spain

E-mail: marcosje@agt.uva.es

Received 11 September 2000;

in revised form 13 December 2001 